\title{
The Establishment and Improvement of Yunnan Regional Economic Multilevel Medical Security System
}

\author{
Youyang You \\ School, \\ Yunnan university of Finance and Economics \\ KunMing, China \\ 85621767@qq.com
}

Regional Economics PhD Graduate Student in School of Public Finance and Economics / Lecturer in International Business

\begin{abstract}
The establishment and improvement of Yunnan regional economic multilevel medical security system, should base on the problems existed in the current Yunnan regional economics. The suggestions should be not only for the multiple needs to the people ,but also for an increase aging population. After all, establish and improve Yunnan multilevel medical insurance system has the necessity and feasibility in Yunnan.
\end{abstract}

Key words--Multilevel; Medical; Security system; Regional economic

\section{INTRODUCTION}

In Yunnan the Twelfth Five-year Plan, the Suggestion of Urban and rural overall planning social security system should be gradually established and improved is proposed, which include improving the basic medical security level, basic medical insurance, medical assistance, complementary medical insurance and the commercial health insurance etc. All of the above belong to the multilevel medical security system. Now the health care reform in China is in an important transition period, establishing a multilevel medical security system under the current national medical security system is inevitable. Establishing multilevel medical security system is a direction for medical insurance system reform afterwards, since single type of medical security system could not support the whole national medical care requirement.
II. MultileVEl MediCAL SECURITY SYSTEM AND ITS FUNCTION.

Multilevel medical security system is based on the basic medical insurance, replenished by Medicaid, Medicaid for civil servants, Enterprise supplementary medical insurance, social medical aid, commercial medical insurance, mutual medical insurance for employees, and the new rural cooperation medical care system. It is an all direction medical security system, and also the most well-run medical security system in China nowadays. The basic thought and main framework of establishing the current multilevel medical security system is "taking the basic social medical insurance as the main body, subsiding by the commercial medical insurance, supporting by the medical aid". Actively establishing the multilevel medical security system could promote the basic medical insurance; meet the needs of different group for the different kind of Medicare consumption. Meanwhile, the multilevel medical insurance system also has the following functions:

\section{A. Ensure the labor health, promote the production}

\section{development}

Medical security is the inevitable outcome of social improvement and production enhancement, the establishment and improvement of medical security could further promote the social improvement and production enhancement. Medical security ensured the labor health both physically and mentally, then further insured re-production, enhance productivity, 
promote production development.

\section{B. Implement the income redistribution, improve the social} fair

Medical security could implement the income re-distribution according to collecting the medical premium and reimbursement the medical service fee; it adjusts the income and life difference between labors, as well as an important social mechanism to adjust social relationship and social confliction. According to this mechanism, the fairness of distribution could be realized in certain degree, and then the unfairness because of market distribution could be offset. In this case, the stability development of social economy is maintained.

\section{Implement the medical security, maintain social stability}

Medical security provides economic aid for the sick labor; alleviate the economic burden because of sickness and maintain their life standards. Moreover, medical security could also eliminate the social unstable factors because of illness, thus, the implements of social medical security system by the government, will benefit the basic medical service of the labors, and benefit the socialism market economic market system and the healthy development of national economy. For all, it benefits the social stability.

\section{Enhance the health consciousness; promote the health} development of hygiene.

The premium sharing mechanism under the medical security system is good for enhancing the consciousness of health care, self-ability for medical security and the consciousness of saving. Since the premium is sharing by the countries, companies and individual, the public medical hygiene service utility is increased, which benefit the healthy development promotion for the hygienic industry. According to improve the needs for the medical service market, the rational distribution for the medical resource is promoted, which guide the medical service market adjusting the service arrangement and service structure, increase service quality, made the medical hygienic industry moving towards a healthy develop way.

\section{THE PROBLEMS EXISTING IN THE YUNNAN REGIONAL} MEDICAL SECURITY SYSTEM

Nowadays, the establishment of Chinese characterized multilevel labor's medical security system is proposed. The current medical security system is constituted by social basic medical insurance, commercial medical insurance, enterprise supplementary medical insurance and rural cooperative medical insurance.

Yunnan is located in the undeveloped South-west region in China; the average income there is comparatively low. But as the development of Yunnan economy, investment for the hygiene service and industry is enhanced fiscally, which provided the foundation for Yunnan medical security physically and technically. Nowadays, the labor insured in Yunnan cities and towns increased annually, the medical fund enlarged continuously; utilization ratio for the new rural cooperative medical service raising money increased, hence, the beneficiary increase further.

But above all, the reform progress for Yunnan medical security development left much to be desired. The main questions for Yunnan medical security system are:

\section{A. Medical security coverage scope structurally unbalanced}

Most of the people in Yunnan is covered by the medical insurance, but from the structure, the main coverage of the basic medical insurance are the labors from the state owned-enterprises and some authority institution, the labors from the other type of institution like part-time worker, vulnerable groups(low-income or laid-off worker, unemployment or disabled, the old and infirm, rural migrant workers, etc.) are not been covered. On the whole, the coverage for Yunnan current medical insurance system is limited, most of the social members do not have the institutional medical coverage. According to the statistics in Yunnan health department, in the overall expenditure of Yunnan hygiene, most of which are the individual expenditure for the medical service, this indicate that the basic national medical security coverage is insufficient.

\section{$B$. The medical hygienic system reform and the medical} insurance system reform unmatched

The medical hygienic system reform is related to the 
establish of basic medical security system. Nowadays, government compensation for the state-owned hospital is less. In order to solve the problems of medical compensation insufficient, the medical institution was allowed to get no less than $15 \%$ of the drug selling price. Driven by the profit pursuit, the hospital normally include expensive implore imported drugs in the prescription. According to the statistics, in the state-owned hospital in Yunnan, the drug selling profit took no less than $40 \%$ of the overall profit. Because of the problems in drug production and drug circulation, artificially high price exists, which seriously affects the consumer's interests. The hospital compensation system of "raising the doctor by medicine", and drug production, circulation system brought strike for the basic medical insurance system.

\section{Medical insurance fund accumulation and expenditure enlarged constantly, and the expenditure increase is larger than the accumulation}

The reason for the large increase of the expenditure than the income in hospital is because of the moral risk. Every 3 years, the incidence of hospitalization which actually contains illness is far less than the incidence of hospitalization., the partial reason is because of the artificially increase of the incidence of hospitalization.

Another reason of the artificial increase in the incidence of hospitalization is because of the individual medical accounts. Take an example of the 2007 Kunming medical center individual accounts, designated retail pharmacies supposed to be the place where the main medical individual accounts should consume, but according to the calculation of illness incidence, the money flowed to the medical expenses supposed less than the money flowed to the designated retail pharmacies. The reason is because of the designated pharmacies provided the other consumption by the individual medical accounts than the medicine. People use the designated pharmacies cash the individual medical accounts.

\section{Irrational differences exists between urban and rural medical security}

Since the urban and rural dual structure in China, there's differences in the urban and rural medical insurance. The urban medical insurance coverage is comparatively higher than the rural medical insurance coverage, and the urban medical service level is higher than the rural medical service level. Compare to the urban medical insurance, the coverage for the new rural cooperation medical is still low. In 2010, the average urban reimbursement ratio for the medical insurance is $75 \%$, but the average rural reimbursement ratio for the new rural medical insurance service is only $50 \%$.

\section{E. The contradiction between the daily increase medical needs and deficiency of medical resource supply.}

In one hand, as the improvement of individual income and enhancement of the personal life, the needs for the medical service is increased too. Those needs not only for the medical service content, but also for the medical scope and level. In the other hand, as the number of labor increased and the aging population increased. The needs for the medical resource is increased too. Comparatively, the supply for the medical service is far less to reach the increasing needs.

\section{SUGGESTIONS TO ESTABLISH THE MULTILEVEL MEDICAL INSURANCE SYSTEM}

In order to establish and improve the medical insurance system, corresponding to Yunnan insurance system, according to the questions above, the suggestions are as the following:

\section{A. Increase the capital investment for the medical service area, provide a solid foundation for the medical insurance system to effectively operation}

The reason why the government should invest the capital is because firstly, increase properly the fiscal aid ratio, could change the operation model of "raising doctors by medicine", then for the other medical insurance system, the government investment could replenish the basic medical insurance.

\section{B. Reform the medical insurance, medical service and} medicine at the same time, create a good environment for the medical insurance system implement.

Firstly, reform the current medical institution, build corresponding medical intuition system. Since the tertiary hospitals is favored by most of the Chinese, the initial diagnose should be designated, two-way referral, then the medical resource could be rationally distributed, which controlled the medical insurance fund expenditure. 
Then, the government should make decisions to resolve totally the problems in the medicine production and circulation, separate the doctor and medicine operation, reverse the situation of artificial high medicine price.

Thirdly, Adjust the supply, need, and insurance interest. Try to develop the medical assist for the vulnerable social group.

C. Develop the community hygienic industry, make a good arrangement of the medical hygienic service system

D. Increase legislation for the social medical insurance, provide a good legislation prove for the medical insurance system

E. Supporting the commercial medical insurance development, forming the real multilevel medical insurance system

F. Pay attention to the establish the labor's serious illness insurance system.

G. Actively build the medical mutual fund and special fund.

H. Establish an scientific medical insurance assessment system, create an sustainable development condition for the medical insurance system

\section{CONCLUSION}

Establish and improve Yunnan multilevel medical insurance system has the necessity and feasibility in Yunnan, not only for the multiple needs to the people ,but also for an increase aging population. The basic medical insurance in China is far less than enough to meet the needs of the people. An multilevel medical insurance system could meet the needs to build a better China.

\section{ACKNOWLEDGEMENT}

Some of the data in this paper were from Dr. Xiao Yugu in Renmin University of China, Dr. Zheng Sujin in Central University of Finance and Economics. Therefore, thanks for the information and helps from these professors.

\section{REFERENCE}

[1] Chen Xiaoming, Medical insurance, Fudan university press.(in Chinese)

[2] Zeng Yu, The new use guide for the health insurance, China building industry press.(in Chinese)

[3] Shi Dexiang, The development of Chinese medical insurance, China price index press.(in Chinese)

[4] KunMing medical insurance center. The report of Kunming medical insurance system operation.(in Chinese)

[5] Zhang Dingqiong. The location of Commercial medical insurance for the multilevel medical security system.(in Chinese) 